Background According to United Nations, 'Philippines has become one of eight countries that account for more than 90 percent of new HIV infections in the region'. (Regencia, 2018) Nonetheless, despite interventions adopted, challenges persist that hinder the efficient implementation of HIV/AIDS program.

Methods The method employed is the Descriptive Correlational Method, a standardized questionnaire on stigma and researcher made questionnaire, to assess level of care and characteristics. Respondents were physicians, nurses, HIV/AIDS Core Team and medical technologists from three different training hospitals in Cagayan de Oro City that underwent Cluster Sampling. Results were statistically analyzed through Spearman Rho for the correlation between characteristics, Stigma and Level of Care and Mean frequency for variables among three training hospitals.

Results Majority of the respondents had an average age of 26 to 30 years old (41.2\%), female (62.2\%), Roman Catholic $(72.5 \%)$ and College graduates $(72.9 \%)$, followed by medical degree holders at $8.4 \%$. Nurses comprised $69.3 \%$ followed by physicians at $9.2 \%$, a majority with an average of one (1) to five (5) years in practice (51.4\%). Religion, profession, workplace and years of experience were significantly correlated with Stigma and years of practice and place of work showed significant relationship with Level of Care. Stigma was inversely correlated with Level of care. Stigma among three hospitals, public and two private hospitals scored average with the highest mean coming from one private hospital at 113.42 compared to a public hospital with a mean of 107.25.

Conclusion It can be determined that there is marked stigma among healthcare workers in Northern Mindanao towards HIV/AIDS patients with a correlate effect on the care and services provided.

Disclosure No significant relationships.

\section{P218 DETERMINING THE NEUROCOGNITIVE STATUS AND FUNCTIONAL ABILITY TO SCREEN FOR HIV-ASSOCIATED NEUROCOGNITIVE DISORDER (HAND)}

${ }^{1}$ Ritika Agarwal* ${ }^{*}$ Ravinder Aujla, ${ }^{1}$ Amit Gupta, ${ }^{2}$ Mukesh Kumar. ${ }^{1}$ Chandra Laxmi Hospital, Medicine, Ghaziabad, India; ${ }^{2}$ Chandra Laxmi Hospital, Ghaziabad, India

\subsection{6/sextrans-2019-sti.362}

Background To adequately evaluate the extent of neurocognitive impairment in PLHIV, a battery of neuropsychological tests is typically administered which are neither cost effective nor time efficient in the outpatient clinical setting. The aim of the study was to assess neurocognitive status and functional ability of people living with HIV and find a brief screening tool to identify those who would benefit from a full diagnostic evaluation.

Methods The study enrolled 160 Patient living with HIV (PLHIV) (80 pre-ART and 80 On-ART) fulfilling the inclusion and exclusion criteria from March 2014 to January 2019. Neurocognitive assessment and an assessment of Functional ability was done by using the Montreal Cognitive Assessment (MoCA) and Lawton and Brody IADL Scale respectively.

Results The study population consisted of $75.6 \%$ males and $24.4 \%$ females with mean age of $44 \pm 10$ years. All of the subjects were literate $(61.2 \%$ subjects had received up to High school level education) and $76.2 \%$ were married. The mean duration of HIV infection among ART naive PLHIV and those on ART was $2 \pm 1.33$ years and $3 \pm 2.10$ years respectively. The overall prevalence of HAND in the study subjects was $52.5 \%$. Of these, $47.5 \%$ had ANI and 5\% had MND. In MoCA, the most frequently affected domains were Language (97.6\%), visuospatial ability (92.9\%) and memory (71.4\%).

Conclusion The prevalence of HAND in both groups were similar suggesting that neurocognitive impairment starts early in HIV infection. Older age ( $>40$ years) and fewer years of formal education were statistically associated with the presence of HAND. HAND involves both cortical and subcortical regions of the brain. Memory and Visuospatial function impairment had the most predictive potential for detecting the presence of HAND. HAND screening is recommended in all PLHIV at enrolment into care. Simple tools like MoCA can be used in busy outpatient settings by healthcare workers to screen for HAND.

Disclosure No significant relationships.

\section{P219 PROFILE OF PATIENTS WHO UNDERWENT RAPID STI TESTS AT A HEALTH UNIT IN THE BRAZILIAN CAPITAL CITY WITH THE HIGHEST HIV RATE}

${ }^{1}$ Ana Amélia Bones*, ${ }^{2}$ Karla Viana, ${ }^{2}$ Érica Duarte, ${ }^{3}$ Luciana Fraga, ${ }^{4}$ Luciane Sebastiany, ${ }^{2}$ Viviane Jost, ${ }^{5}$ Luciana Teixeira. ${ }^{1}$ Health Federal University from Porto Alegre, Health Science Post Graduation Program, Porto Alegre, Brazil; ${ }^{2}$ Federal University of Rio Grande do Sul, Nursing School, Porto Alegre, Brazil; ${ }^{3}$ Health Municipal Secretary from Porto Alegre, Porto Alegre, Brazil; ${ }^{4}$ Municipal Health Secretary from Porto Alegre, Porto Alegre, Brazil; ${ }^{5}$ Federal University of Rio Grande do Sul, Collective Health Graduation, Porto Alegre, Brazil

\subsection{6/sextrans-2019-sti.363}

Background From 1980 to June 2018, 926,742 cases of AIDS were identified in Brazil, with an average of 40,000 new cases in the five years. Porto Alegre, the capital of the state of Rio Grande do Sul in the south of Brazil, presented a rate of 60.8 cases/100 thousand inhabitants in 2017, more than twice the rate of the state as a whole and 3.3 times higher than the overall Brazilian rate. The guarantee of treatment for all in 2013 contributed to the drop in rates, in addition to expanding access to testing and reducing the time from diagnosis to the start of treatment. The aim here is to evaluate the profile of the patients who underwent a rapid test for HIV and other STIs offered at a primary health care unit in Porto Alegre.

Methods An observational, cross-sectional epidemiological study with six years of collection was completed in December 2018 for the study. Profile differences between patients with positive and negative results were examined for the tests they underwent. The level of significance was $5 \%$.

Results Of the 1717 patients tested, 68.7\%(n =1164) were women, 33\%(n =385) pregnant, 9.4\%(n =140) with more than 12 years education, $3.3 \%$ illiterate, and $4.7 \%$ street dwellers. Of the total, 7.4\% ( $\mathrm{n}=122)$ were HIV positive, $2.8 \%(\mathrm{n}=39)$ for hepatitis $\mathrm{C}, 0.8 \%(\mathrm{n}=9)$ for hepatitis $\mathrm{B}$, $15.6 \%(\mathrm{n}=267)$ syphilis reagents, and $1.4 \%(\mathrm{n}=23)$ had tuberculosis. Compared with sociodemographic variables, there was a statistical difference in relation to HIV, with a higher proportion of women ( $\mathrm{p}=0.003)$, and a lower educational level $(\mathrm{p}=0.02)$.

Conclusion The results showed a vulnerability to HIV infection related to socioeconomic issues and the female sex. Gestation appears as an opportune time for diagnosis since rapid test should be taken in the first trimester, the third trimester, and at the time of delivery.

Disclosure No significant relationships. 